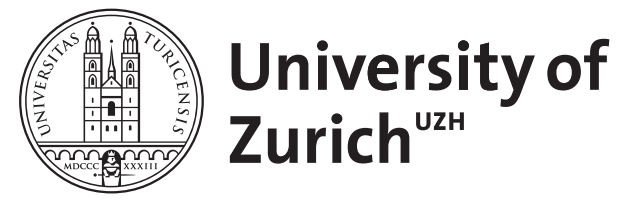

Zurich Open Repository and Archive

University of Zurich

University Library

Strickhofstrasse 39

CH-8057 Zurich

www.zora.uzh.ch

Year: 2012

"Enjoy a delicious cake today and eat healthily tomorrow": Compensatory Health Beliefs and their impact on health

Radtke, Theda ; Scholz, Urte

Posted at the Zurich Open Repository and Archive, University of Zurich

ZORA URL: https://doi.org/10.5167/uzh-97762

Journal Article

Published Version

Originally published at:

Radtke, Theda; Scholz, Urte (2012). "Enjoy a delicious cake today and eat healthily tomorrow": Compensatory Health Beliefs and their impact on health. The European Health Psychologist, 14(2):37-40. 


\section{'Enjoy a delicious cake today and eat healthily tomorrow': Compensatory Health Beliefs and their impact on health}

\section{Theda Radtke \& Urte Scholz}

University of Konstanz
Every day we are faced with temptations like drinking a beer after finishing work or eating a delicious cake. In such situations the immediate desire to indulge in the pleasant behaviour like eating the cake can interfere with our longterm goals (e.g., be healthy or lose weight). According to Muraven and Baumeister (2000) humans strive to achieve an ideal balance between the completion of their desires and the pursuit of their own goals. This search of the ideal balance between maximum pleasure and minimal disadvantage is called the hedonic principle. However, the interaction between our desire and our health goals can lead to a motivational conflict (Rabiau, Knäuper, \& Miquelon, 2006), or so-called cognitive dissonance (Festinger, 1957), because of the incompatibility between both goals. This dissonance generates a state of pressure, whose resolution requires self-regulatory processes to deal with the aversive state of dissonance (Rabiau et al., 2006). According to the Compensatory Health Belief (CHB) Model one possible strategy to diminish this conflict is to use/employ Compensatory Health Beliefs (Knäuper, Rabiau, Cohen, \& Patriciu, 2004).

CHBs are beliefs that the negative effects of an unhealthy (but pleasurable) behaviour can be compensated for or neutralised by carrying out a healthy behaviour (Rabiau et al., 2006, p. 140). To resolve the cognitive dissonance, people convince themselves that eating the cake or drinking a beer is ok because they exercise the following day, or they eat healthily and this behaviour will compensate for the negative effects of alcohol or fatty food. Thus, CHBs seem to provide an ideal solution, since they allow us e.g., to eat unhealthily without feeling guilty about having counteracted our own goals. The activation of CHBs is an automatically motivated regulatory process to reduce cognitive dissonance, by justifying unhealthy behaviour with future planned healthy behaviour, as mentioned by Knäuper and colleagues (2004). Likewise, it seems also reasonable that the planned unhealthy behaviour in the future is cognitively neutralised by a healthy behaviour an individual already engaged in in the past. Thus, CHBs can be activated before or after the behaviour has been carried out. According to the CHB model, CHBs are activated when there is a high self-concordance of the health goals, and if the person fails to keep up the aspired goal (Knäuper et al., 2004; Kronick \& Knäuper, 2010).

\section{CHBs: good effects on dissonance, negative effects on health}

According to Rabiau and colleagues (2006), CHBs can be correct, partly correct, or incorrect. The classification can be difficult, because oftentimes unhealthy behaviour causes several negative effects on health and the compensatory behaviour only compensates for some but not all adverse effects (cf., Knäuper et al., 2004). An example for a partial compensation is that the risk of developing cancer, which is elevated by smoking, can potentially be buffered but not completely neutralized by a healthy nutrition (Kuper, Adami, \& Bofetta, 2002). Moreover, it is not guaranteed that people actually carry out the intended compensatory behaviour (Knäuper 
et al., 2004), because too much time may have passed by between the activation of the CHBs and the planned implementation of the compensatory behaviour. Thus, the dissonant feeling and the necessity to compensate the unhealthy behaviour fades away. Consequently, CHBs interfere with successful adherence to health behaviour changes such as dieting or quitting drinking alcohol.

\section{Overview of empirical results concerning CHBs}

First evidence for the relatively new construct of CHBs comes from Knäuper et al. (2004) who developed a scale to measure CHBs in general. They found that CHBs are positively associated with health-related risk behaviours like alcohol consumption or smoking and with symptom reports. Further research also indicates that CHBs are associated with lower goal achievement (Rabiau, Knäuper, Nguyen, Sufrategui, \& Polychronakos, 2009). Results have shown that adolescents, who had been diagnosed with type 1 diabetes, hold compensatory beliefs concerning their glucose testing. As shown, these beliefs were associated with less regular testing of the glucose level and with poorer metabolic control. In line with this research, Nguyen, Knäuper, and Rabiau (2006) found that the more CHBs diabetic adolescents held, the less likely they were to control their glycemic level, monitor their bloodsugar, and adhere to their dieting rules. Furthermore, Monson, Knäuper, and Kronick (2008) showed that dieters spontaneously generate CHBs in response to temptation. This is in line with results from a study by Kronick and Knäuper (2010), which found that dieters had compensatory intentions on their mind when they were faced with the food temptation of a delicious cookie. In addition, the authors found support for the proposition that the existence of compensatory intentions is related to the decision to eat the high caloric cookie. It seems that compensatory intentions are one strategy to cope with temptations when individuals allow themselves to indulge. Another study of a sample of coronary heart disease patients also revealed that nutrition style is best explained by CHBs in addition to self-efficacy (Taut \& Baban, 2008). However, it is important to mention that in this study no other variables except for selfefficacy and CHBs were entered in the analysis.

\section{CHBs in comparison to other psychological constructs}

Even though the results mentioned above lead to the assumption that CHBs can be considered to be an important factor in unsuccessful self-regulation regarding health behaviour, none of the reported studies investigated $\mathrm{CHBs}$ in comparison to other health-psychological constructs. Therefore, and because the CHB model (Rabiau et al., 2006) mainly focuses on explaining the generation of CHBs, but not on the association of CHBs with intention formation and behavioural change, we conducted a study with adolescent smokers to investigate the CHBs within the framework of a theoretical model (Radtke, Scholz, Keller, \& Hornung, in press).

Since results regarding the general CHB scale from Knäuper and colleagues (2004) indicate an unstable factor structure across different countries (Kaklamanou \& Armitage, in press; Radtke, Scholz, Keller, Perren, \& Hornung, 2011), we first developed a new smoking-specific CHB scale to overcome problems of the factor structure and to improve the matching on levels of specificity (Radtke, Scholz, Keller, Knäuper, \& Hornung, 2011). This newly developed scale could be identified as relevant with regard to the readiness to change smoking patterns in adolescents (Radtke et al., 2011). Subsequently, we examined the added value of CHBs over and above factors of a health behaviour change model: the Health Action Process Approach (HAPA; Schwarzer, 2008). The sample consisted 
of 224 adolescent smokers who filled in an online-questionnaire. All HAPA-specific variables like self-efficacy, outcome expectancies, risk awareness, intention to stop smoking, planning and smoking behaviour as well as the smokingspecific CHBs were assessed. In line with previous research mentioned above, we found that smoking-specific CHBs were significantly negatively associated with the intention to stop smoking over and above HAPA-specific predictors. However, no direct association between smoking-specific CHBs and smoking behaviour was found. Overall, CHBs provide a very promising construct to explain why individuals often fail to generate and/or to follow their intentions. Yet, as always in the case of a rather new construct, quite a number of unanswered questions remain.

\section{Future research challenges}

Overall, more studies are needed to investigate the impact of CHBs on intention or behaviour. First of all, we need more research on everyday and on longer-term effects of CHBs for health behaviour change. Focusing on the everyday perspective, the question would be in which situations and how often CHBs are used during a day and how this relates to (antecedents of) behaviour. Moreover, it should be explored whether CHBs are activated before (as a result of anticipated guilt) or after the performance of unhealthy behaviour. Another key question that deserves future attention is the investigation whether there is a difference between beliefs of compensatory health behaviour and the execution of them, because CHBs are first and foremost a cognitive strategy ('belief') and need to be differentiated from compensatory behaviour. First results of Kaklamanou, Armitage, and Jones (2012) yielded evidence for the assumption that individuals distinguish between the belief and the behaviour. This means that individuals on the one hand behave in a way that is consistent with the CHBs, but on the other hand do not really believe in the compensational effect. Moreover, research focusing on $\mathrm{CHBs}$ in interventions is strongly needed. How can CHBs be overcome or how can their impact be effectively reduced?

\section{Conclusion}

As the research summarized above demonstrated, CHBs are a potential barrier of behaviour change in different health domains (e.g., dieting or smoking), because the justification of eating a delicious cake by compensating for it later undermines people's intentions and goal achievement to behave healthily. Therefore, CHBs offer the potential to enhance the effectiveness of behaviour change interventions in the future.

\section{References}

Festinger, L. (1957). A Theory of Cognitive Dissonance. Stanford, CA: Stanford University Press.

Kaklamanou, D., \& Armitage, C. J. (in press). Testing compensatory health beliefs in a UK population. Psychology and Health.

Kaklamanou, D., Armitage C. J., \& Jones, C. R. (2012). A further look into compensatory health beliefs. Manuscript submitted for publication.

Knäuper, B., Rabiau, M., Cohen, 0., \& Patriciu, N. (2004). Compensatory health beliefs scale development and psychometric properties. Psychology and Health, 19, 607-624. doi:10.1080/0887044042000196737

Kronick I., \& Knäuper, B. (2010). Temptations elicit compensatory intentions. Appetite, 54, 398-401. doi:10.1016/j.appet.2009.12.011

Kuper, H., Adami, H.-0., \& Boffetta, P. (2002). Tobacco use, cancer causation and public health impact. Journal of Internal Medicine, 251, 455-466. doi:10.1046/j.1365$2796.2002 .00993 . x$

Monson, E., Knäuper, B., \& Kronick, I. (2008). Food temptations spontaneously elicit compensatory beliefs in dieters. McGill Science Undergrad Research Journal, 3, 42-45.

Muraven, M., \& Baumeister, R. F. (2000). Self-regulation 
and depletion of limited resources: Does self-control resemble a muscle? Psychological Bulletin, 126, 247-259. doi:10.1.1.112.8019

Nguyen, T.-K., Knäuper, B., \& Rabiau, M. (2006). The role of compensatory beliefs and self-efficacy on treatment adherence in adolescents with type 1 diabetes. McGill Science Undergrad Research Journal, 1, 7-10. doi:10.1093/her/cyp032

Rabiau, M., Knäuper, B., \& Miquelon, P. (2006). The eternal quest for optimal balance between maximizing pleasure and minimizing harm: The Compensatory Health Beliefs model. British Journal of Health Psychology, 11, 139153. doi:10.1348/135910705X52237

Rabiau, M., Knäuper, B., Nguyen, T.-K., Sufrategui, M., \& Polychronakos, C. (2009). Compensatory beliefs about glucose testing are associated with low adherence to treatment and poor metabolic control in adolescents with type 1 diabetes. Health Education Research, 24, 890-896. doi:10.1093/her/cyp032

Radtke, T., Scholz, U., Keller, R., \& Hornung, R. (in press). Smoking is ok as long as I eat healthily: Compensatory Health Beliefs and their role for intentions and smoking within the Health Action Process Approach. Psychology and Health.

Radtke, T., Scholz, U., Keller, R., Knäuper, B., \& Hornung, R. (2011). Smoking-specific Compensatory Health Beliefs and the readiness to stop smoking in adolescents. British Journal of Health Psychology, 16, 610-625. doi:10.1348/2044-8287.002001
Radtke, T., Scholz, U., Keller, R., Perren, S., \& Hornung, R. (2011). Assessing the psychometric properties of the German version of the Compensatory Health Belief Scale. Manuscript submitted for publication.

Schwarzer, R. (2008). Modeling health behavior change: How to predict and modify the adoption and maintenance of health behaviors. Applied Psychology: An International Review, 57, 1-29. doi:10.1111/j.14640597.2007.00325.x

Taut, D., \& Baban, A. (2008). Examination of the relationship between self-regulation strategies and healthy eating patterns in coronary heart disease patients. The relevance of Compensatory Health Beliefs. Cognition, Brain \& Behavior, 12, 219-231.

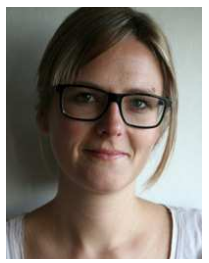

Theda Radtke

is Postdoc at the Department of Developmental and Health Psychology, University of Konstanz, Germany

\section{Theda.Radtke@uni-konstanz.de}

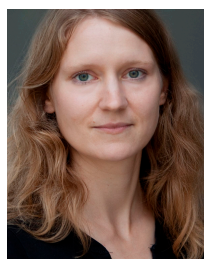

\section{Urte Scholz}

is Professor of Developmental and Health Psychology at the University of Konstanz, Germany 